\title{
Correlation between Radiological Staging of Chronic Rhinosinusitis and Revision of Endoscopic Sinus Surgery
}

\author{
Dhaidan Alshammari' ${ }^{1}$, Raneem Alshaikh ${ }^{2}$, Manuel Bakheet ${ }^{3}$, Waleed Janahi' ${ }^{2}$, Hiba Alreefy ${ }^{4}$ \\ ${ }^{1}$ Ministry of Health, Riyadh, KSA \\ ${ }^{2}$ King Hamad University Hospital, Busaiteen, Kingdom of Bahrain \\ ${ }^{3}$ Ministry of Health, Manama, Kingdom of Bahrain \\ ${ }^{4}$ Awali Hospital, Awali, Kingdom of Bahrain \\ Email: dhaidanmohsen1411@hotmail.com,dr.r.alshaikh@hotmal.com, manuel.zaki@hotmail.com,waleed.janahi@khuh.org.bh, \\ drhibaalreefy@hotmail.com
}

How to cite this paper: Alshammari, D., Alshaikh, R., Bakheet, M., Janahi, W. and Alreefy, H. (2021) Correlation between Radiological Staging of Chronic Rhinosinusitis and Revision of Endoscopic Sinus Surgery. International Journal of Otolaryngology and Head \& Neck Surgery, 10, 119-130. https://doi.org/10.4236/ijohns.2021.102013

Received: February 22, 2021

Accepted: March 28, 2021

Published: March 31, 2021

Copyright $\odot 2021$ by author(s) and Scientific Research Publishing Inc. This work is licensed under the Creative Commons Attribution International License (CC BY 4.0).

http://creativecommons.org/licenses/by/4.0/

(c) (i) Open Access

\begin{abstract}
Introduction: Chronic rhinosinusitis (CRS) is an inflammatory condition affecting the sinonasal mucosa with duration of more than 12 weeks. Multifactorial aetiology contributes to its refractoriness. Functional endoscopic sinus surgery (FESS) is the most common surgical technique preferred in CRS not responding to medical management, showing high success rates. However, some failure has been noted, requiring revision FESS. CT (Computed Tomography) is a diagnostic tool for CRS, and aids in improving surgical outcomes. Combined with the Lund-Mackay scoring system, CT can help stage the CRS, predicting the need for revision surgery. Methods: In our paper, we aimed to study the relationship between severity of CRS using radiological staging and revision of functional endoscopic sinus surgery, assessing outcome after at least 1 year of follow-up. There was a single centre retrospective study involving 150 patients selected by random sampling, to study the correlation between the severity of chronic rhinosinusitis and revision of functional endoscopic sinus surgery (FESS). The study was conducted in the ENT department of King Hamad University Hospital, Bahrain for a time period of 6 months. Records were scanned and scored using Lund-Mackay system. Lund Mackay score of 10 and above was considered severe. The relation between increased severity with Lund Mackay score on the CT scan and the need for revision sinus surgeries was then determined. Results and Conclusion: This study concludes that LMS score in CT scans can be used to predict the outcomes of primary FESS procedure and the need for revision surgery in the management of CRS.
\end{abstract}




\section{Keywords}

FESS, Lund-Mackay System, ENT, Revision Sinus Surgery, Revision FESS, Chronic Rhinosinusitis (CRS)

\section{Introduction}

Chronic rhinosinusitis (CRS) is an inflammatory condition affecting the sinonasal mucosa [1]. Duration should last for more than 12 weeks to categorize it as CRS. Most times, CRS is refractory to medical management [2]. Symptoms can vary between nasal obstructions, facial heaviness to complete anosmia. The aetiology of chronic rhinosinusitis is largely unknown but is likely to be multifactorial, with inflammation, infection and obstruction of sinus ventilation playing a part [3] [4]. Some epidemiologic grounds of association have been established between the prevalence of CRS and air pollution, cigarette smoking (active and passive), perennial allergic rhinitis and gastroesophageal reflux [5].

CRS is a significant condition affecting around $14 \%$ of the adult population in the US [6]. Clinical findings include: nasal polyps, oedema of the nasal mucosa and purulent discharge. Radiologic investigations using computed tomography (CT) of the paranasal sinuses can show mucosal thickening with opacifications of air cells [7].

A common problem seen in the ENT clinics, CRS has a worldwide prevalence of $5 \%-12 \%$, seen frequently in the urban populations [8]. The majority of patients with CRS (both paediatric and adult age group) are, in fact, immune competent [5]. As it has a significant effect on the quality of life, individuals seek medical help with a desperate need for relief.

Treatment is attempted primarily by medical therapy, while surgery is preserved for patient with persistent symptoms. Medical therapy includes oral steroids, intranasal steroids and saline irrigations. This treatment should be given for trial duration of 6 weeks before deciding on any surgical intervention. Amongst various modalities, Functional endoscopic sinus surgery (FESS) has become one of the most popular surgical techniques performed by otolaryngologists as treatment for CRS over the last three decades. Significant data demonstrates the efficacy of FESS in combination with appropriate medical treatments and good post-operative treatments [9] [10] [11].

Recent studies [12] suggested that early FESS in patients with mild CT scoring can help in improving surgical outcomes. However, despite the exceptional published success rates of $75 \%$ - 98\%, given the contributory factors, some patients with CRS without nasal polyposis may present with recurrent symptoms and revision surgery is a requirement in nearly $10 \%-15 \%$ [13] [14]. Many factors attribute to failure of FESS, including inappropriate patient selection, inadequate patient preparation, severe mucosal disease, staging of sinus disease, anatomical variations that have not been identified or adequately addressed, inferior surgical skills and deficient postoperative care [13] [14]. 
Revision functional endoscopic sinus surgery (revision FESS) poses a challenge to the otolaryngologist, requiring a meticulous preoperative review of patient's anatomy, re-assessment of the severity of the disease, and also, the underlying comorbidities contributing to the symptoms. CT scan and nasal endoscopy hold reputation as the best modalities to assess the anatomical variation and areas of scarring obstructing the natural ostium of each sinus.

The objective of this study is to assess the outcome of FESS, chronicled according to the severity of CRS, after at least 1 year of follow-up. The study also aims to further evaluate the various risk factors (e.g. age, comorbidities, and compliance to medications) that can reflect on the morbidity associated with chronic rhinosinusitis and help in improving the outcome of management.

Chronic rhinosinusitis management can be arduous, given the variety of contributing factors (anatomical, environmental and patient's compliance) affecting it, all of which can influence the success rates. Although CRS is a widely-spread condition encountered by otorhinolaryngologists in Bahrain, there are no clear statistics indicating its prevalence in the country. The implications of this study are that it can highlight the shortcomings in the surgical procedure, thereby helping to improve outcomes of surgery.

\section{Literature Review}

CRS (Chronic Rhinosinusitis) is characterized by persistent nasal and paranasal mucosal inflammation. Rosenfield et al., 2015, updated the clinical guidelines for adult sinusitis, refining the criteria to diagnose CRS [15]. The requirement of empirical evidence of inflammation, observed clinically, was established as an important component. Chronic Rhinosinusitis was defined as "inflammation of paranasal sinuses and nasal cavity lasting more than 12 weeks, with or without acute exacerbations" [15].

Different classification systems are used to stage the severity of sinus disease including Lund Mackay scoring system [13]. This is a radiological scoring, modified by Okashi et al. in 2013, to give the Modified Lund Mackay scoring system, done to each side separately according to the extent of inflammation/opacification in each sinus. Maximum score is $24.0-3$ will be considered mild, while $4-10$ is moderate, and more than 10 is severe. The table below (Table 1) elaborates the scoring system:

Table 1. The Lund-Mackey scoring system.

\begin{tabular}{ccc}
\hline SINUS & RIGHT SINUS & LEFT SINUS \\
\hline FRONTAL & $0-2$ & $0-2$ \\
ANTERIOR ETHMOIDS & $0-2$ & $0-2$ \\
POSTERIOR ETHMOIDS & $0-2$ & $0-2$ \\
MAXILLAR & $0-2$ & $0-2$ \\
SPHENOID & $0-2$ & $0-2$ \\
OSTIOMEATAL COMPLEX & 0 or 2 & 0 or 2 \\
\hline
\end{tabular}

For the sinuses: $0=$ no inflammation; $1=$ partial inflammation; $2=100 \%$ inflammation; For the ostiomeatal complex: 0 = not occluded; 2 = occluded; Maximum total score: 24 . 
Amongst surgical modalities, especially in refractive rhinosinusitis, FESS has been the treatment of choice with high success rates. Damm et al. (2002) established that the outcomes with FESS proved it an adequate treatment for CRS, particularly in combination with good postoperative medical care [16]. Patel et al. (2017), reinforced the improvement in health utility and olfaction in medically refractory CRS, as well as the cost-effectiveness of using FESS compared to sole continuation of medical therapy [17]. Although in paediatric age group, patients who underwent Balloon Catheter Sinuplasty for CRS required significantly fewer antibiotics post-operatively related disease when compared to FESS [11], major and minor complications following paediatric FESS rate as low as $0.6 \%$, and $2 \%$ respectively, with a positive outcome ranging between $71 \%$ and $100 \%$ [18].

Failure of FESS, although, is not uncommon. The factors mostly contributing to failure include inappropriate techniques, lack of proper assessment and deficient post-operative care. Any plan for revision surgery must meticulously address the anatomic factors contributing to recurrence. A systematic review of the preoperative imaging and sinonasal endoscopy is necessary; areas of disease and involvement of "danger" zone (which is the triangular area between the angles of the mouth and nasal bridge) are especially identified, as traditional anatomic landmarks are often obscured or absent. A set of consistent landmarks, i.e. those that remain unchanged despite prior surgery, are thus used to navigate the revision FESS [14].

Persistent chronic sinusitis or recurrent infections in a patient despite primary sinus surgery has a need for aggressive treatment with antibiotics and steroids. If symptoms persist, a C.T (Computed tomography) scan is essential to identify the source of infection, revision surgery indicated once the anatomic aetiology of primary failure is identified [19]. Chang et al. claimed that FESS combined with concurrent SMR/septoplasty had less incidence of revision rate of FESS [20].

Computed tomography (CT) has gained popularity as the most commonly used imaging modality for evaluation of CRS. Sakuma et al. [21] and Zuo et al. [22] ascertained that CT characteristics could be used in diagnosing eosinophilic CRS (ECRS). With their relatively low cost, ease of storage, and low-dose radiation exposure, CT scans, especially Point of Care-CT (PoC-CT), have become an indispensable tool in the management of CRS [23]. In the setting of increasing antibiotic costs, overtreatment with antibiotics, and fewer required return visits, PoC-CT challenges the conventional role of empiric medical therapy before progression to imaging for the diagnosis of CRS. Amodu et al. identified that CT scan scores could help predict severity of symptoms regarding nasal obstruction and discharge, but not for other symptoms of chronic rhinosinusitis. Overall disease severity scores also showed no association with CT score [24].

An evaluation study using the Sinonasal Assessment Questionnaire (SNAQ) and Lund-Mackay (LM) grading system found that although no statistically significant correlation existed between SNAQ and LM scores, the scoring of scans by the surgeon and the radiologist seem to have strong associations. Morbidity 
of patients with CRS could also not be predicted from the magnitude of changes seen in their CT scans [7]. In about $60 \%-70 \%$ of cases, the CT scan grading can be found correlating with operative findings. However, though CT provides a detailed picture of sinus involvement, its relation with symptom severity cannot be relied upon. Compared to the Lund-McKay CT staging (LMCTS) system, the Kennedy CT staging system seemed to correlate better with CRS symptoms [25]. Brooks et al. established that LMCTS is indeed an indicator of postsurgical QoL outcomes [26], which can further be developed to predict the need for revision surgeries, especially in medically recalcitrant chronic rhinosinusitis patients.

CRS, thus, can be seen to have a substantial impact on the quality of life of an individual. Patients with CRS have health utility scores worse than most chronic diseases, including many cardiac ailments and COPD (Chronic Obstructive Pulmonary Disorder) [27] [28]. Treatment of CRS can reduce the fatigue and bodily pain substantially, improve the health state utility scores of patients and their quality of life [16] [18] [27] [28] [29]. Treatment should aim to minimize recurrence of CRS, keeping in mind the multifactorial aetiology requiring revision FESS, ranging from severe mucosal disease to anatomical variations not addressed precisely in primary surgery [2]. Techniques to predict the possible need for revision surgery are being explored, in order to improve the primary procedure as well as anticipate the necessity in future.

\section{Methodology}

A retrospective study was attempted with prior approval from the research committee, in a single centre setting, to study the correlation between the severity of chronic rhinosinusitis based on radiological staging using Lund-Mackay scoring and revision of functional endoscopic sinus surgery (FESS). The study was conducted in the ENT department of King Hamad University Hospital, Bahrain. All adult patients (above the age of 16) who underwent functional endoscopic sinus surgery in the institute from 2015-2020 by a single surgeon were included in the study. The diagnosis included allergic rhinosinusitis with or without nasal polyposis and fungal rhinosinusitis.

Patients who underwent FESS for antrochoanal polyps or sinus tumours as well as patients who underwent FESS outside the hospital were excluded from the study. Those who did not have CT scan in our system were also excluded.

Considering these factors, hospital database (HOPE system at our hospital) was used to access patient records (using surgical lists) of 185 patients were reviewed using random sampling technique. The time period of the study was 6 months, during which data was collected and studied. The information extracted from the records included Patient demographics (age > 16), Comorbidities (e.g. diabetes, asthma), Duration of trial of medical therapy (at least 6 weeks), Primary sinus surgery and Revisions (if any). CT scan for each patient was reviewed and scored using Lund Mackay scoring system (please find attached copy).

As stated earlier, Lund Mackay score of 10 and above is considered severe. The relation between increased severity of Lund Mackay and the need for revi- 
sion sinus surgeries was then analysed and duly summarized using SPSS software. Variables such as demographic data, the LM-CTS scores and the number of revision FESS surgeries conducted were collected. The data obtained was preserved in a well-locked cabinet in the clinic with access only by the investigators involved in this research.

\section{Results}

The study aims to elucidate the relation between radiological staging of CRS and its outcome on FESS.

The study population included 185 adult patients with symptoms of CRS, of which 81 were females and 104 were males. Maximal cases (approx. 69\%) of exclusive chronic rhinosinusitis of unknown aetiology were seen, these included patients with septal deviation and concha bullosa, while the next frequent diagnosis (approx. 26\%) was chronic rhinosinusitis with nasal polyposis. Others were fungal rhinosinusitis (4.3\%). Of the total study group, 55 (29.7\%) patients required revision procedure. Figure 1 shows the frequency of revision cases under each diagnosis categories.

The Lund-Mackay CT staging scores were employed to determine the severity of CRS. A score > 10 was considered as a "severe" case of CRS. Table 2 categorizes the frequency of cases who required revision surgeries based on their LMCTS scores. Majority of the total participants $(n=185)$ scored $>10(n=116$, $62.7 \%)$, while a lesser share of $37.3 \%(n=69)$ patients scored $<10$.

63 out of $69(91.3 \%)$ patients who had scores $<10$ did not require a revision procedure, while in those having scores $>10$, only $57.8 \%$ did not require the revision procedure. The odds of revision procedure could, thus, be estimated to be about eight times higher in the patients with LMCTS $>10$ as compared to the patients in the group LMCTS $<10(\mathrm{OR}=7.69,95 \%$ CI: $3.07-19.17, \mathrm{p}<0.000)$.

Table 3 shows the LMCT mean score comparison between revision and non-revision group, which was examined using bivariate analysis. It was found that there exists a positive correlation between the LMCT scores and the need of revision procedure $(\mathrm{r}=0.464, \mathrm{p}<0.00$, two tailed), i.e., higher the scores, the need of revision is higher.

Comorbidities: Out of 18 patients who had "asthma", 12 required revision procedure. The odds of performing a revision procedure was higher among asthma patients compared to patients with other comorbidities (HTN, hyperthyroidism, DM) $(\mathrm{OR}=5.78,95 \% \mathrm{CI}: 2.03-16.31, \mathrm{p}=\mathbf{0 . 0 0 1})$.

Table 2. Frequency of revision surgery according to LMCTS scores.

\begin{tabular}{cccc}
\hline & No Revision & Revision & Total \\
\hline LMCTS $<10$ & $63(91.3 \%)$ & $6(8.7 \%)$ & 69 \\
LMCTS $>10$ & $67(57.8 \%)$ & $49(42.2 \%)$ & 116 \\
Total & $130(70.3 \%)$ & $55(29.7 \%)$ & 185 \\
\hline
\end{tabular}

Chi Square $\mathrm{p}<0.000$ 
Table 3. LMCT mean score comparison between revision and non-revision group:

\begin{tabular}{ccc}
\hline & Mean \pm SD (LMCTS) & P value \\
\hline Revision & $16.83 \pm 4.57$ & $<0.005$ \\
No revision & $11.82 \pm 4.19$ & \\
\hline
\end{tabular}

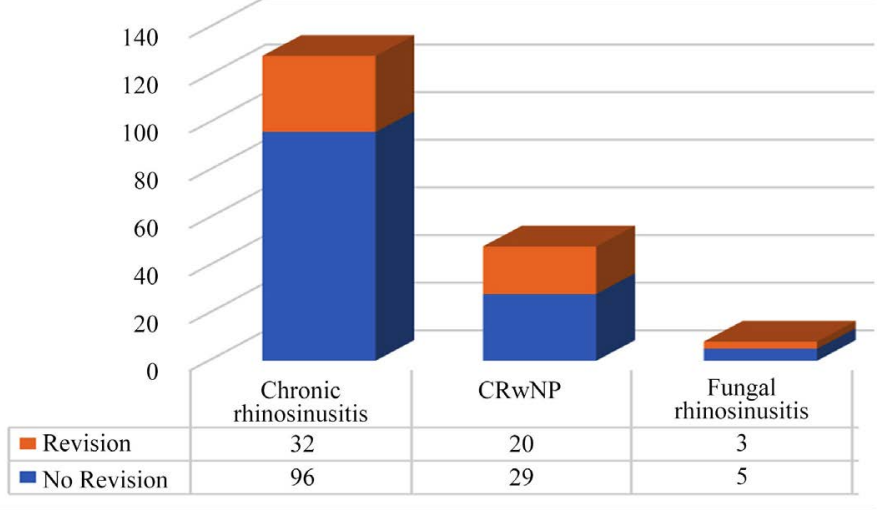

Figure 1. Frequency of revision cases under each diagnosis category.

\section{Discussion}

Recurrence of CRS requiring a revision FESS procedure is tiresome from both the patient and the physician perspective. Patients with CRS per se, experience many physical, psychosocial and financial grievances, with the hope to be relieved by surgery. As CRS diagnosis is based on subjective and objective criteria, it is difficult to predict the outcomes of management. To this day, there is no fool-proof treatment for CRS: the diagnosis, treatment and outcome management remain, as yet, controversial.

Physicians evaluate CRS outcomes using different instruments. Objective evaluation can be done effectively using Computed Tomography (CT) scores, nasal endoscopy as well as QOL scores after surgical management [16] [17] [18] [30] [31]. Correlation of scores on patient-based questionnaires and objective scales like CT and endoscopy have also been significant [4] [32] [33] [34]. The majority of participants in the present study were male, as seen in similar studies by Khalil [19] and Zheng [4]. The gender variation does not seem to have any importance or influence in the course of the disease or its management.

A key difficulty in the management of chronic rhinosinusitis is that despite the reported success rates, quite a few patients report varying degrees of symptom persistence. Many patients require long-term continuation of medical management. The degree of improvement of symptoms post-procedure is a common query asked by every patient party. The answer, however, is indeterminate, as each individual responds differently to the FESS procedure. The quest to identify or create a predictive tool, which can allow physicians to predict the need for a secondary procedure, can, not only allow for earlier revision and relief of ailment, but also improve facets of the primary procedure, bringing to light 
many overlooked parameters.

The accuracy and validity of CT scans in CRS staging are well established, the Lund-MacKay system showing the strongest linear relationship between CT stage and sinonasal symptoms amongst the staging instruments [23] [32] [35]. The LM staging system is simple and quite effective, grading the symptom severity on a scale of 1 - 10, with each sinus being graded separately on the basis of whether it is clear, partially opacified, or totally opacified, and thus, considered the best tool for this study [25] [36] [37]. Ashraf and Bhattacharya proposed that a mean LMS score of 4 was required to perform revision FESS, Hopkins et al. identified a mean score of 7 and a study by Socher had a mean score of 5.71 [6] [38] [39]. The present study obtained a mean of 16.83 (SD: 4.57) in the LMS score for those patients requiring revision FESS. Congruent to Socher, the population submitted to revision here was also homogenous.

Preoperative LMCTS scores were significantly associated with postoperative SNOT-22 scores for CRS in a study by Brooks et al., 2018 [26]. At 12 months, patients with lower pre-op LMCTS had lesser QoL outcomes, which in terms of revision procedure requirements, could mean a possibility of needing revision FESS in the near future. Although limited to the extranasal and rhinologic domains of the SNOT-22, these are key in predicting the possibility of failure of the primary procedure.

Comorbidities in patients with CRS, can be identified by specific questions regarding history of previous treatments, current medications, social habits and radiographic imaging as well. Compared to other co-morbid conditions, the prevalence of asthma was found higher in the group with recurrence as compared to other comorbidities in the present study. Yifan et al., 2019 also established that prevalence of asthma was higher in the recurrence group [40]. A study by Matsuwaki in 2008 also recorded higher prevalence of asthma in the group showing recurrence of symptoms [3]. Thus, it can be considered as a prime risk factor for revision procedure in the pre-operative evaluation itself, allowing for more intensive management protocols to be framed for treatment.

\section{Limitations}

The study does not attempt to investigate the clinical outcome in the patients after revision surgery, keeping to the aim of the study which was to assess the outcome of FESS at minimally 1 year of follow up and the factors that may result in recurrent rhinosinusitis after primary surgery, reflecting on the practice of FESS. The bias in sampling may limit the study, and the small sample size may make generalization of findings difficult.

\section{Conclusion}

Analysis of the CT scans in patients with recurrent CRS, requiring revision surgery can help in the identification of factors such as persistent anatomic structures that may be responsible. Despite the development of various treatment 
strategies, recurrence in CRS still presents a major challenge in its management. This study concludes that an LMS score in CT scans is predictive of the need for revision surgery, i.e. the higher the score, the greater the need for revision surgery. Also, patients having asthma as comorbidity show higher rates of recurrence as compared to others. The present study hopes to stimulate further studies to compare CT scores in their diagnostic and predictive value, and to answer the question of how extensive sinus surgery should be.

\section{Conflicts of Interest}

The authors declare no conflicts of interest regarding the publication of this paper.

\section{References}

[1] Benninger, M.S., Ferguson, B.J., Hadley, J.A., Hamilos, D.L., Jacobs, M., Kennedy, D.W., et al. (2003) Adult Chronic Rhinosinusitis: Definitions, Diagnosis, Epidemiology, and Pathophysiology. Otolaryngology-Head and Neck Surgery, 129, S1-S32. https://doi.org/10.1016\%2FS0194-5998\%2803\%2901397-4

[2] Baban, M.I.A., Mirza, B. and Castelnuovo, P. (2020) Radiological and Endoscopic Findings in Patients Undergoing Revision Endoscopic Sinus Surgery. Surgical and Radiologic Anatomy, 42, 1003-1012. https://pubmed.ncbi.nlm.nih.gov/32025784/ https://doi.org/10.1007/s00276-020-02427-5

[3] Matsuwaki, Y., Ookushi, T., Asaka, D., Mori, E., Nakajima, T., Yoshida, T., et al. (2008) Chronic Rhinosinusitis: Risk Factors for the Recurrence of Chronic Rhinosinusitis Based on 5-Year Follow-Up after Endoscopic Sinus Surgery. International Archives of Allergy and Immunology, 146, 77-81.

https://doi.org/10.1159/000126066

[4] Zheng, Y.B., Zhao, Y., Lv, D., Liu, Y.F., Qiao, X.M., An, P., et al. (2010) Correlation between Computed Tomography Staging and Quality of Life Instruments in $\mathrm{Pa}$ tients with Chronic Rhinosinusitis. American Journal of Rhinology \& Allergy, 24, e41-e45. https://doi.org/10.2500/ajra.2010.24.3430

[5] Hamilos, D.L. (2011) Chronic Rhinosinusitis: Epidemiology and Medical Management. Journal of Allergy and Clinical Immunology, 128, 693-707. https://doi.org/10.1016/j.jaci.2011.08.004

[6] Socher, J.A., Mello, J. and Baltha, B.B. (2018) Tomographical Findings in Adult Patients Undergoing Endoscopic Sinus Surgery Revision. International Archives of Otorhinolaryngology, 22, 73-80. https://doi.org/10.1055/s-0037-1601417

[7] Basu, S., Georgalas, C., Kumar, B.N. and Desai, S. (2005) Correlation between Symptoms and Radiological Findings in Patients with Chronic Rhinosinusitis: An Evaluation Study Using the Sinonasal Assessment Questionnaire and Lund-Mackay Grading System. European Archives of Oto-Rhino-Laryngology and Head \& Neck, 262, 751-754. https://doi.org/10.1007/s00405-004-0891-0

[8] Dietz de Loos, D., Lourijsen, E.S., Wildeman, M.A.M., Freling, N.J.M., Wolvers, M.D.J., Reitsma, S., et al. (2019) Prevalence of Chronic Rhinosinusitis in the General Population Based on Sinus Radiology and Symptomatology. Journal of Allergy and Clinical Immunology, 143, 1207-1214.

https://doi.org/10.1016/j.jaci.2018.12.986

https://pubmed.ncbi.nlm.nih.gov/30578880/ 
[9] Siedek, V., Stelter, K., Betz, C.S., Berghaus, A. and Leunig, A. (2009) Functional Endoscopic Sinus Surgery-A Retrospective Analysis of 115 Children and Adolescents with Chronic Rhinosinusitis. International Journal of Pediatric Otorhinolaryngology, 73, 741-745. https://doi.org/10.1016/j.ijporl.2009.01.019

[10] van der Veen, J., Seys, S.F., Timmermans, M., Levie, P., Jorissen, M., Fokkens, W.J., et al. (2017) Real-Life Study Showing Uncontrolled Rhinosinusitis after Sinus Surgery in a Tertiary Referral Centre. Allergy, 72, 282-290. https://doi.org/10.1111/all.12983

[11] Thottam, P.J., Haupert, M., Saraiya, S., Dworkin, J., Sirigiri, R. and Belenky, W.M. (2012) Functional Endoscopic Sinus Surgery (FESS) Alone versus Balloon Catheter Sinuplasty (BCS) and Ethmoidectomy: A Comparative Outcome Analysis in Pediatric Chronic Rhinosinusitis. International Journal of Pediatric Otorhinolaryngology, 76, 1355-1360. https://doi.org/10.1016/j.ijporl.2012.06.006

[12] Rudmik, L., MacE, J. and Smith, T. (2011) Low-Stage Computed Tomography Chronic Rhinosinusitis: What Is the Role of Endoscopic Sinus Surgery? Laryngoscope, 121, 417-421. https://doi.org/10.1002/lary.21382

[13] Okushi, T., Nakayama, T., Morimoto, S., Arai, C., Omura, K., Asaka, D., et al. (2013) A Modified Lund-Mackay System for Radiological Evaluation of Chronic Rhinosinusitis. Auris Nasus Larynx, 40, 548-553.

https://doi.org/10.1016/j.anl.2013.04.010

[14] Levine, C.G. and Casiano, R.R. (2017) Revision Functional Endoscopic Sinus Surgery. Otolaryngologic Clinics of North America, 50, 143-164.

https://doi.org/10.1016/j.otc.2016.08.012

[15] Rosenfeld, R.M., Piccirillo, J.F., Chandrasekhar, S.S., Brook, I., Ashok Kumar, K., Kramper, M., et al. (2015) Clinical Practice Guideline (Update): Adult Sinusitis Executive Summary. Otolaryngology-Head and Neck Surgery, 152, 598-609. https://doi.org/10.1177/0194599815574247

[16] Damm, M., Quante, G., Jungehuelsing, M. and Stennert, E. (2002) Impact of Functional Endoscopic Sinus Surgery on Symptoms and Quality of Life in Chronic Rhinosinusitis. Laryngoscope, 112, 310-315. https://doi.org/10.1097/00005537-200202000-00020

[17] Patel, Z.M., Thamboo, A., Rudmik, L., Nayak, J.V., Smith, T.L. and Hwang, P.H. (2017) Surgical Therapy vs Continued Medical Therapy for Medically Refractory Chronic Rhinosinusitis: A Systematic Review and Meta-Analysis. International Forum of Allergy \& Rhinology, 7, 119-127. https://doi.org/10.1002/alr.21872

[18] Vlastarakos, P.V., Fetta, M., Segas, J.V., Maragoudakis, P. and Nikolopoulos, T.P. (2013) Functional Endoscopic Sinus Surgery Improves Sinus-Related Symptoms and Quality of Life in Children with Chronic Rhinosinusitis: A Systematic Analysis and Meta-Analysis of Published Interventional Studies. Clinical Pediatrics, 52, 1091-1097. https://doi.org/10.1177/0009922813506489

[19] Khalil, H.S., Eweiss, A.Z. and Clifton, N. (2011) Radiological Findings in Patients Undergoing Revision Endoscopic Sinus Surgery: A Retrospective Case Series Study. BMC Ear, Nose and Throat Disorders, 11, Article No. 4. https://doi.org/10.1186/1472-6815-11-4

[20] Chang, C.C., Tai, C.J., Ng, T.Y., Tsou, Y.A. and Tsai, M.H. (2014) Can FESS Combined with Submucosal Resection (SMR)/Septoplasty Reduce Revision Rate? Otolaryngology-Head and Neck Surgery, 151, 700-705. https://doi.org/10.1177/0194599814543778

[21] Sakuma, Y., Ishitoya, J., Komatsu, M., Shiono, O., Hirama, M., Yamashita, Y., et al. 
(2011) New Clinical Diagnostic Criteria for Eosinophilic Chronic Rhinosinusitis. Auris Nasus Larynx, 38, 583-588. https://doi.org/10.1016/j.anl.2011.01.007

[22] Zuo, K., Guo, J., Chen, F., Xu, R., Xu, G., Shi, J., et al. (2014) Clinical Characteristics and Surrogate Markers of Eosinophilic Chronic Rhinosinusitis in Southern China. European Archives of Oto-Rhino-Laryngology, 271, 2461-2468.

https://doi.org/10.1007/s00405-014-2910-0

[23] Leung, R., Chaung, K., Kelly, J.L. and Chandra, R.K. (2011) Advancements in Computed Tomography Management of Chronic Rhinosinusitis. American Journal of Rhinology \& Allergy, 25, 299-302. https://doi.org/10.2500/ajra.2011.25.3641

[24] Amodu, E.J., Fasunla, A.J., Akano, A.O. and Olusesi, A.D. (2014) Chronic Rhinosinusitis: Correlation of Symptoms with Computed Tomography Scan Findings. Pan African Medical Journal, 18, Article No. 40.

https://doi.org/10.11604/pamj.2014.18.40.2839

[25] Rathor, A. and Bhattacharjee, A. (2017) Clinical-Radiological Correlation and Role of Computed Tomography Staging in Chronic Rhinosinusitis. World Journal of Otorhinolaryngology-Head and Neck Surgery, 3, 169-175.

https://doi.org/10.1016/j.wjorl.2017.02.008

[26] Brooks, S.G., Trope, M., Blasetti, M., Doghramji, L., Parasher, A., Glicksman, J.T., et al. (2018) Preoperative Lund-Mackay Computed Tomography Score Is Associated with Preoperative Symptom Severity and Predicts Quality-of-Life Outcome Trajectories after Sinus Surgery. International Forum of Allergy \& Rhinology, 8, 668-675. https://doi.org/10.1002/alr.22109

[27] Luk, L.J., Steele, T.O., Mace, J.C., Soler, Z.M., Rudmik, L. and Smith, T.L. (2015) Health Utility Outcomes in Patients Undergoing Medical Management for Chronic Rhinosinusitis: A Prospective Multiinstitutional Study. International Forum of Allergy \& Rhinology, 5, 1018-1027. https://doi.org/10.1002/alr.21588

[28] Soler, Z.M., Wittenberg, E., Schlosser, R.J., MacE, J.C. and Smith, T.L. (2011) Health State Utility Values in Patients Undergoing Endoscopic Sinus Surgery. Laryngoscope, 121, 2672-2678. https://doi.org/10.1002/lary.21847

[29] Chester, A.C., Sindwani, R., Smith, T.L. and Bhattacharyya, N. (2008) Fatigue Improvement Following Endoscopic Sinus Surgery: A Systematic Review and Meta-Analysis. Laryngoscope, 118, 730-739.

[30] Poetker, D.M., Mendolia-Loffredo, S. and Smith, T.L. (2007) Outcomes of Endoscopic Sinus Surgery for Chronic Rhinosinusitis Associated with Sinonasal Polyposis. American Journal of Rhinology \& Allergy, 21, 84-88. https://doi.org/10.2500/ajr.2007.21.2978

[31] Soler, Z.M. and Smith, T.L. (2010) Quality of Life Outcomes after Functional Endoscopic Sinus Surgery. Otolaryngologic Clinics of North America, 43, 605-612. https://doi.org/10.1016/j.otc.2010.03.001

[32] Bhattacharyya, N. (2005) A Comparison of Symptom Scores and Radiographic Staging Systems in Chronic Rhinosinusitis. American Journal of Rhinology \& Allergy, 19, 175-179. https://doi.org/10.1177/194589240501900210

[33] Wabnitz, D.A.M., Nair, S. and Wormald, P.J. (2005) Correlation between Preoperative Symptom Scores, Quality-of-Life Questionnaires, and Staging with Computed Tomography in Patients with Chronic Rhinosinusitis. American Journal of Rhinology \& Allergy, 19, 91-96. https://doi.org/10.1177/194589240501900116

[34] Smith, T.L., Rhee, J.S., Loehrl, T.A., Burzynski, M.L., Laud, P.W. and Nattinger, A.B. (2003) Objective Testing and Quality-of-Life Evaluation in Surgical Candidates with Chronic Rhinosinusitis. American Journal of Rhinology \& Allergy, 17, 351-356. 
https://doi.org/10.1177/194589240301700607

[35] Bhattacharyya, N. and Fried, M.P. (2003) The Accuracy of Computed Tomography in the Diagnosis of Chronic Rhinosinusitis. Laryngoscope, 113, 125-129.

https://doi.org/10.1097/00005537-200301000-00023

[36] Bhattacharyya, N. (2006) Radiographic Stage Fails to Predict Symptom Outcomes after Endoscopic Sinus Surgery for Chronic Rhinosinusitis. Laryngoscope, 116, 18-22. https://doi.org/10.1097/01.mlg.0000192284.22703.04

[37] Rhyoo, C., Jung, M.K. and Lee, J.H. (2016) The Clinical Significance of Lund-Mackay CT Staging System in Assessing the Severity of Chronic Rhinosinusitis. Korean Journal of Otorhinolaryngology-Head and Neck Surgery, 44, 837-841.

http://www.koreamed.org/SearchBasic.php?RID=2275756

[38] Ashraf, N. and Bhahacharyya, N. (2001) Determination of the "Incidental" Lund Score for the Staging of Chronic Rhinosinusitis. Otolaryngology-Head and Neck Surgery, 125, 483-486. https://doi.org/10.1067/mhn.2001.119324

[39] Hopkins, C., Browne, J.P., Slack, R., Lund, V. and Brown, P. (2007) The Lund-Mackay Staging System for Chronic Rhinosinusitis: How Is it Used and What Does It Predict? Otolaryngology-Head and Neck Surgery, 137, 555-561.

https://doi.org/10.1016/j.otohns.2007.02.004

[40] Meng, Y., Zhang, L., Lou, H. and Wang, C. (2019) Predictive Value of Computed Tomography in the Recurrence of Chronic Rhinosinusitis with Nasal Polyps. International Forum of Allergy \& Rhinology, 9, 1236-1243.

https://doi.org/10.1002/alr.22355 